\title{
Excision of Orbital Dermoid Cysts via Upper Eyelid Incision: A Review of 24 Cases
}

\author{
Rustu Kose
}

\begin{abstract}
Background: Dermoid cysts are abnormally located masses arising from abnormal involution of superficial ectoderm during closure of sutures between bones. Dermoid cysts are the most common orbital masses in childhood. They should be surgically removed at ages 1 - 2 as they may enlarge and lead to deformation in orbital bony structures and erosion. Traditional treatment is removal of the mass via an incision over the mass. Incisions through, below or above the brow are used to remove these cysts. Endoscopic incision of these cysts is also performed.
\end{abstract}

Methods: In this study, 24 patients aged between 2 and 18 years underwent dermoid cyst excision via upper eyelid incision.

Results: Cyst rupture did not occur except two patients. Mean operative time was found as 20 minutes. Loss of vision or eyelid functions occurred in no patients. Cyst recurrence was observed in no patients. All patients or parents were satisfied with the scar.

Discussion: There is not a significant difference between direct incision over the mass and upper eyelid incision in terms of cyst rupture and operative time. As upper eyelid skin is the region of the body that hides the scar best with its thin skin, these masses may be removed easily and with a good field of vision via upper eyelid incision.

Keywords: Dermoid cysts; Upper eyelid incision; Excision

\section{Introduction}

Dermoid cysts are abnormally located masses arising from abnormal involution of superficial ectoderm during closure of sutures between bones. These cysts are composed of keratinized stratified squamous epithelium, have a fibrous capsule,

Manuscript accepted for publication August 18, 2014

Department of Plastic Reconstructive and Esthetic Surgery, Medical Faculty, Recep Tayyip Erdogan University, Rize, Turkey.

Email: rustu.kose@erdogan.edu.tr

doi: http://dx.doi.org/10.14740/jcs240w and also have skin appendices like sweat glands, sebaceous glands and hair follicles [1].

Dermoid cysts are the most common orbital masses in childhood [2]. They consist of $40 \%$ of childhood orbital lesions, $89 \%$ of childhood orbital cysts [3]. Most cysts are present at birth or in the first 3 months of life [4]. Dermoid cysts in orbita are located at zygomaticofrontal suture line in the ratio of $86.6 \%$, nasofrontal suture line in the ratio of $10 \%$. Patients usually apply doctors with complaints of mass $(100 \%)$ or proptosis $(10 \%)$ [5]. Inflammatory reaction in surrounding tissues as the result of leakage of cyst content may rarely be the reason for admission. They should be surgically removed at ages $1-2$ as they may enlarge and lead to deformation in orbital bony structures and erosion [6]. Traditional treatment is removal of the mass via an incision over the mass. Incisions through, below or above the brow are used to remove these cysts. Endoscopic incision of these cysts is also performed.

In this study, 24 patients underwent orbital dermoid cyst excision via an upper eyelid incision. Scarring tissue, operative time, complications and recurrence results were evaluated.

\section{Methods and Results}

In this study, 24 patients aged between 2 and 18 years (mean age 11) underwent dermoid cyst excision via upper eyelid incision. Cysts were left-sided in 17 cases and right-sided in seven cases. All patients were performed computed tomography preoperatively in order to determine the presence of intracranial extension of cysts and orbital bony defect. Pomade was applied to the eye prior to the operation in order to protect eyeball. Borders of the cyst and incision site were marked preoperatively (Fig. 1). Incisions were first made $1 \mathrm{~cm}$ in size and $1 \mathrm{~mm}$ above supratarsal line, extended about $1.5 \mathrm{~cm}$ when needed intraoperatively. Cyst was accessed by proceding above the brow following skin incision (Fig. 2). Cyst was accessed by incising the muscle (Fig. 3). Most of the cysts were seen to be adjacent and above the periosteum. Some cysts were below the periosteum. Complete excision of cysts was made together with periosteum using periosteum elevator (Fig. 4). Cyst rupture did not occur except two patients. Cyst content was removed by washing with saline solution in patients with cyst rupture. Hemorrhage control was done using thin ended bipolar cautery. Orbicularis muscle was closed with intermit- 


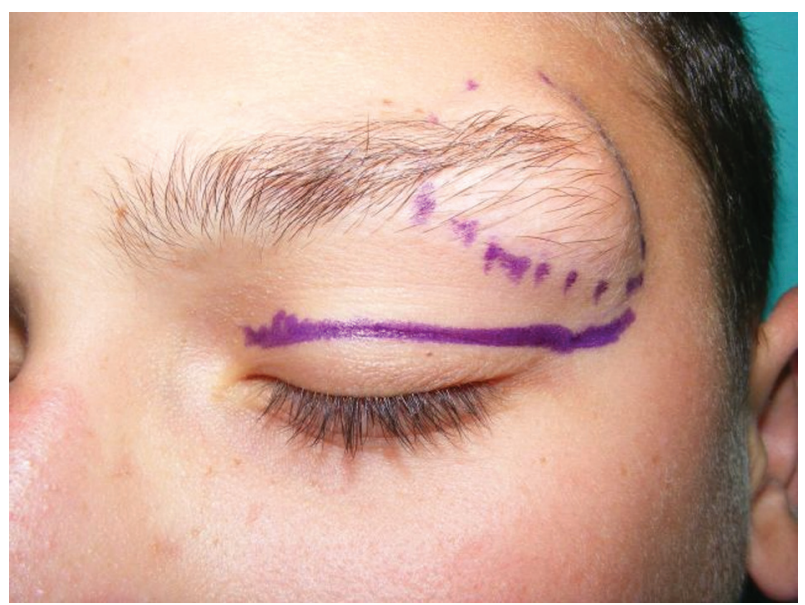

Figure 1. Marking of dermoid and eyelid crease.

tent 6-0 monocryl suture, skin was closed with 6-0 polypropylene suture intracutaneously and steri-strips were applied. Mean operative time was found as $20 \mathrm{~min}$. Patients were followed up for 3 - 24 months (mean 10 months). Loss of vision or eyelid functions occurred in no patients. Cyst recurrence was observed in no patients. All patients or parents were satisfied with the scar. Scar was not perceptible especially when the eye is open.

\section{Discussion}

Purpose of orbital dermoid cyst treatment is cyst removal with minimal dissection and maximum concealing. Obtaining an esthetic and functional outcome after surgery is also an important principle in dermoid cyst surgery besides complete removal of the mass.

Traditional treatment is excision of the mass with an incision over the mass. After this incision, long-standing erythema and sometimes a bad looking sunk scar may remain due to thickness of the skin in this area. Scarring occurs more in children as the skin is tight in children [7]. Incisions through, below or above the brow are used to remove these cysts. Although the scar is hidden in the brow in this incision, alope-

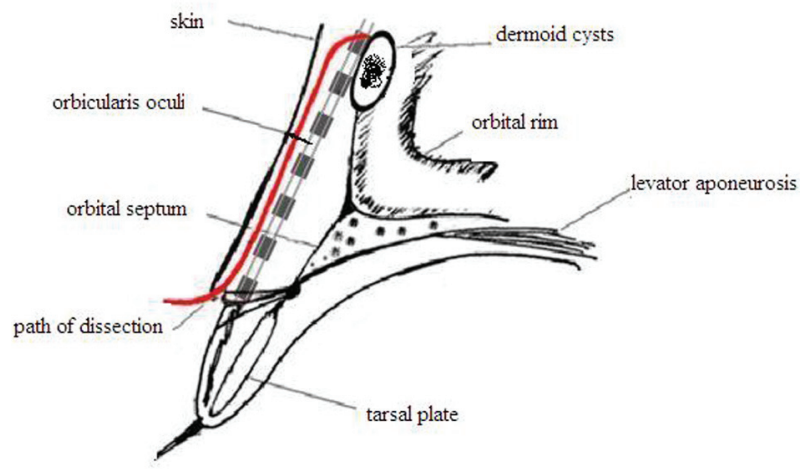

Figure 2. Plane of dissection fol-

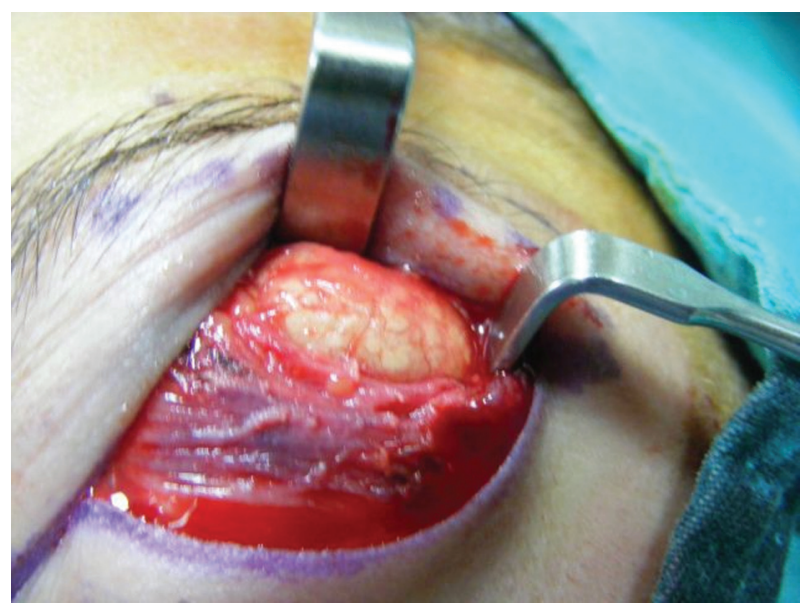

Figure 3. Cyst was accessed by incising the muscle.

cia and deformity may develop as brow follicles are oblique. These cysts may endoscopically be removed [8]. Special devices and experience are needed for this excision. Injury may develop in frontal branch of fascial nerve [9]. The best option for cyst extending into frontal sinus or cranium is bicoronal incision. Upper eyelid incision for orbital dermoid cysts was first used by Kertsen in 1988 [2]. Although this incision is not routinely used today, it has begun to be preferred more frequently recently due to good scar $[6-8,10]$.

Marking cyst borders and incision site preoperatively provide convenience for the surgeon. Eye should be closed with pomade in order to protect eyeball and a suspension suture should be placed. Upper eyelid incision is made $1 \mathrm{~mm}$ above supratarsal line. Incision should be $1-1.5 \mathrm{~cm}$ in size depending on the size and location of the cyst. Dissection may be done in two ways following incision. While cyst may be accessed over orbital septum by opening orbicularis muscle [8], muscle may be opened here when the mass is exposed through a dissection between the skin and the muscle (Fig. 2). We accessed the cyst between skin and muscle in all cases. So we avoided complications like injuring lavator aponeurosis, lacrimal gland or supe-

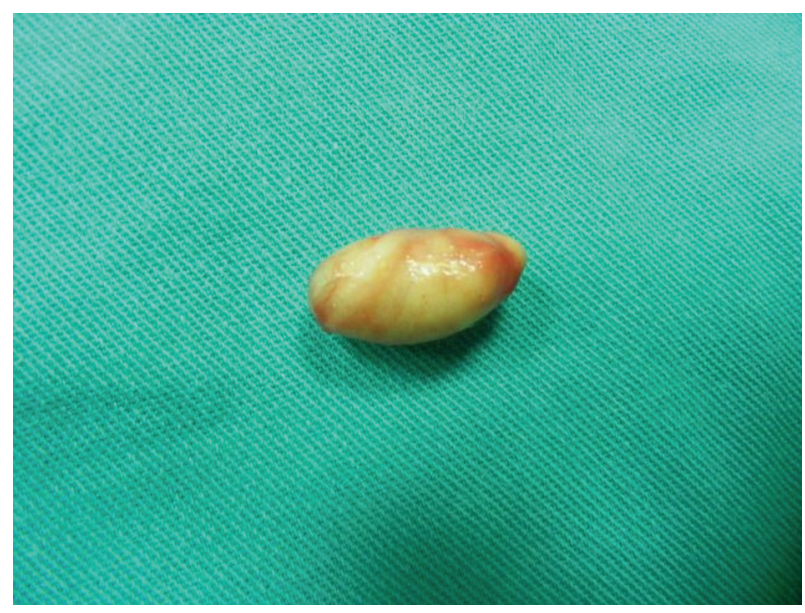

Figure 4. Complete excision of dermoid cysts. 
rior oblique muscle tendon by passing through septum orbitale. Cyst rupture is a complication that should be avoided most but its occurrence is possible. Cyst content should be removed from wound site by washing with abundant saline solution.

Although dermoid cysts are congenital lesions, time of admission to doctor and operation may vary. While cysts may be located superficially on periosteum, they may be located deeply under periosteum. Deeply located cysts are recognized late and when they become larger. Although their removal is recommended until age 2 , time of operation is around 12 years in many studies [9]. Mean operation age was 11 in our study. Bony erosions in late removed lesions improve with time. Studies are also available reporting that these masses may be operated in earlier ages $[6,10]$.

In our study, needed time to remove these cysts was 20 min. In similar case serials, time to remove the cysts with incision over the mass was found as $25 \mathrm{~min}$ [11] and time for endoscopic removal was found as $50.5 \mathrm{~min}$ [12]. We considered that short operative time in our study was related with the fact that the surgeon had gained experience, all patients were operated by him, most of the cysts were located on the periosteum and none of the cysts had intracranial or intraorbital extensions. This duration was reported as average $21 \mathrm{~min}$ in a study including 54 cases, and the same incision was used [10].

When evaluated in terms of cyst rupture, while studies are available reporting that rates are the same with others in upper eyelid incision [7], studies are also available reporting that no rupture had occurred [10]. According to our opinion, there is not a correlation between incision and cyst rupture.

Computed tomography should be performed preoperatively in order to determine the presence of intracranial extension of cysts and orbital bone defect. In our study, all patients were performed computed tomography preoperatively. Intracranial or intraorbital extension was detected in no patients. Distinguishing dermoid cysts from diseases like dacryoadenitis, lacrimal canal cyst, dacryocele, mucocele, capillary hemangioma, orbital abscess, preseptal orbital cellulitis preoperatively is especially of importance. Magnetic resonance imaging is beneficial for detection of cyst content and differential diagnosis.

\section{Conclusion}

There is not a significant difference between direct incision over the mass and upper eyelid incision in terms of cyst rupture and operative time. As upper eyelid skin is the region of the body that hides the scar best with its thin skin, these masses may be removed easily and with a good field of vision via upper eyelid incision.

\section{References}

1. Pryor SG, Lewis JE, Weaver AL, Orvidas LJ. Pediatric dermoid cysts of the head and neck. Otolaryngol Head Neck Surg. 2005;132(6):938-942.

2. Kersten RC. The eyelid crease approach to superficial lateral dermoid cysts. J Pediatr Ophthalmol Strabismus. 1988;25(1):48-51.

3. Shields JA, Shields CL. Orbital cysts of childhood--classification, clinical features, and management. Surv Ophthalmol. 2004;49(3):281-299.

4. Doxanas MT, Green R, Arensten JJ, Elsas FJ. Lid lesions of childhood: a histopathologic survey at the Wilmer Institute (1923-1974). J Pediatr Ophthalmol. 1976;13:7-39.

5. Cavazza S, Laffi GL, Lodi L, Gasparrini E, Tassinari G. Orbital dermoid cyst of childhood: clinical pathologic findings, classification and management. Int Ophthalmol. 2011;31(2):93-97.

6. Nelson KE, Mishra A, Duncan C. Upper blepharoplasty approach to frontozygomatic dermoid cysts. J Craniofac Surg. 2011;22(6):e41-44.

7. Kose R, Okur MI. Comparison of superior eyelid incision and directly over the lesion incision to brow dermoid cyst excision. Eur J Plast Surg. 2009;32:83-85.

8. Park AH, Siddiqi F. An approach to pediatric brow dermoids: an upper eyelid crease incision. Int J Pediatr Otorhinolaryngol. 2006;70(2):349-351.

9. Lane CM, Ehrlich WW, Wright JE. Orbital dermoid cyst. Eye (Lond). 1987;1 (Pt 4):504-511.

10. Ruszkowski A, Caouette-Laberge L, Bortoluzzi P, Egerszegi EP. Superior eyelid incision: an alternative approach for frontozygomatic dermoid cyst excision. Ann Plast Surg. 2000;44(6):591-594; discussion 594-595.

11. Gur E, Drielsma R, Thomson HG. Angular dermoid cysts in the endoscopic era: retrospective analysis of aesthetic results using the direct, classic method. Plast Reconstr Surg. 2004;113(5):1324-1329.

12. Steele MH, Suskind DL, Moses M, Kluka E, Liu DC. Orbitofacial masses in children: an endoscopic approach. Arch Otolaryngol Head Neck Surg. 2002;128(4):409413. 